\title{
The Self-Growing Concept as a Design Principle of Cognitive Self-Organization
}

\author{
Marc Emmelmann $^{l}$, B. Bochow ${ }^{l}$, Athanasios Makris ${ }^{2}$, Alexandros Kaloxylos ${ }^{2 \dagger}$, Georgios P. Koudouridis ${ }^{3}$ \\ ${ }^{1}$ Fraunhofer-Gesellschaft zur Förderung der angewandten Forschung e.V., Germany- \\ \{bernd.bochow,marc.emmelmann\}@fokus.fraunhofer.de \\ ${ }^{2}$ National and Kapodistrian University of Athens, Greece - \{tmakris, agk\}@di.uoa.gr \\ ${ }^{\dagger}$ Department of Telecommunications Science \& Technology, University of Peloponnese, Tripolis, Greece - kaloxyl@uop.gr \\ ${ }^{3}$ Huawei Technologies R\&D Center, Sweden-george.koudouridis@huawei.com
}

\begin{abstract}
In next generation systems and networks selforganization in networks of collaborating networks is expected to relax some of the intricacies of managing complex cooperative communication systems. In particular, in the presence of distributed cognitive decision-making, increasing complexity may increase potential interference between collaborating networks hence leading to performance, robustness and dependability issues. This paper focuses on a specific form of self-organization denoted here as self-growing, which is believed to provide a foundation for flexible, open and trustworthy networks, relax some of the scalability issues of collaborating cognitive networks, as well as to enable selforganization for resource constrained systems.

Keywords: self-growing; energy-awareness; purpose-driven; lifecycle; collaborating networks; distributed cognitive decisionmaking
\end{abstract}

\section{INTRODUCTION}

Self-organization of networks of collaborating networks is believed to provide a means to resolve some of the intricacies in maintaining performance, robustness and dependability when faced with changes in network utilization and topology, while (or due to) responding and adapting to varying user requirements. In particular, heterogeneous networks that individually apply self-x features relying on distributed decision-making may introduce an unforeseeable complexity and potential of interference in a collaboration, which in turn may cause severe scalability issues. On the other hand complex selforganization may result in a significant control and management overhead that restricts its applicability to resource-constrained systems such as wireless mobile or sensor networks that have to balance energy consumption, emitted RF power, shared medium access and achievable data rate.

The self-growing paradigm [3] is presented here as a design principle for self-organization of collaborating networks utilizing configurable, heterogeneous nodes and networks, and distributed cognitive engines. As a specialized form of self-organization it is believed to relax some of the scalability issues and to enable self-organization for resource constrained systems.

This paper first describes the objectives of the EU FP7 CONSERN project which aims to develop and validate a new paradigm for cooperating and energy-aware networks denoted as self-growing.
After introducing the terminology used throughout this paper, section III concentrates on the concept of purposes and lifecycles in the context of self-growing networks. It provides a very basic description of how lifecycles can be generated, evaluated and optimized, mainly referencing the underlying project's work for more details.

Section IV then describes the cognitive architecture assumed to realize a self-growing network, while section V discusses selected use cases which are assumed to emphasize the benefit of applying the self-growing paradigm to certain problems of reconfiguring and recovering from a reconfiguration of a network of collaborating networks.

Concluding, section VI is summarizing the key aspects of self-growing, highlighting in that the expected benefit of applying the self-growing paradigm to future networks from an operator's perspective.

\section{CONSERN PROJECT OBJECTIVES}

As systems get more complex in terms of scale, functionality and heterogeneity, reliability and dependability are getting increasingly important and self-adaptation techniques for achieving dependable system operation under cost and energy constraints will be a key concept. Low energy consumption, efficient use of energy and energy harvesting are considered fundamental enablers in the efforts to combat climate change and to achieve sustainable economic growth. In particular, for wireless and mobile systems low RF emission, interference avoidance, coexistence and incumbent protection is crucial for the efficient use of frequency spectrum as a scarce natural resource.

In this context the CONSERN project [1][2] is addressing cooperative and energy-aware networks aiming to develop and validate a novel paradigm denoted as a selfgrowing network.

A 'self-growing network' is considered a novel type of network composed of (heterogeneous) network nodes and sub-networks that can cooperate and utilize their reconfiguration capacity to optimize on-demand for a dedicated (temporary) purpose, also augmenting capacity by associating with additional nodes, networks, services and functions in that. In contrast to conventional self-organizing networks, a self-growing network is purpose-driven and is following a predetermined or dynamically adjusted lifecycle. Herein a 'purpose' is considered an abstract description of the intended use of a collaboration of networks defined by a 
set of parameters and metrics. It may be expressed as a set of well-described configuration parameters a certain collection of nodes and networks can assume. Thus, the term 'purposedriven' in this context highlights that the evolution of a selfgrowing network is following a certain path between attainable purposes guided by a rule set. A (well-determined) set of purposes and the network's progression along these purposes defines the 'lifecycle' of a self-growing network. Since purposes may change due to external events, even well-defined and (at deployment time) pre-determined lifecycles are subject to optimization with respect to resources availability, such as the remaining battery power of a network node.

The CONSERN project aims to validate this approach by considering two distinct types of scenarios and use cases targeting a network reconfiguration due to a purpose change:

- Use cases that reflect a current business case such as reconfiguring a wireless network to optimize network offloading for certain types of traffic, including sophisticated use cases such as M2M communication in a dynamic collaboration of wireless sensor networks and collocated access networks;

- Use cases that reflect new applications in vertical markets such as responding to an incident by an immediate and substantial reconfiguration of the wireless network topology and functionality within the affected geographical area, including issues such as how to recover from such a reconfiguration and maintaining as much as possible from the original network capacity when returning to normal operations.

Suitable methods of validating the concept (e.g. test cases and evaluation metrics) are currently studied from a perspective that may allow applying the very same methodology to post-deployment optimization. For example, a test case and performance evaluation metric applied offline could also be used to determine cost and benefit of a purpose change (i.e. to predict the result of a configuration change) if a network has to decide when and how it should collaborate with another collocated network.

\section{Design PRINCIPLES FOR PURPOSES AND LifECYCle}

At the beginning of its lifecycle (Figure III-1), defined here as the progression through a series of differing stages of development which can potentially provide different services, a self-growing network is set up on-demand, dedicated to a single purpose. In the course of its lifecycle a self-growing network may coexist and cooperate with other wireless networks of distinct owners or interest groups evolving in the deployment area towards using or augmenting existing capacity. Towards the end of its lifecycle, the self-growing network may still remain active and serve, for example, as a dedicated purpose network or as a failover for applications associated with other networks sharing the same area.

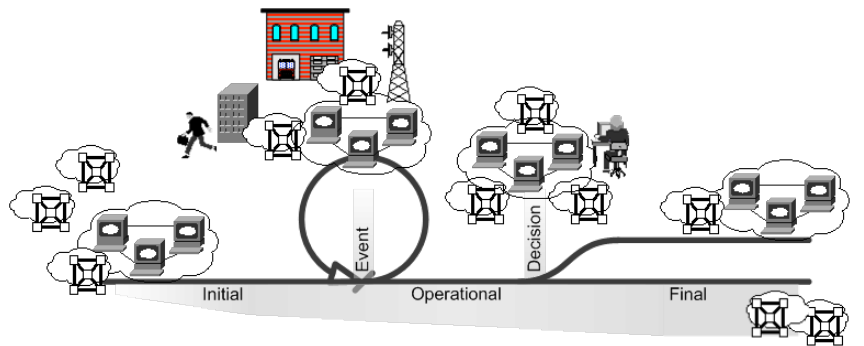

Figure III-1: Sample self-growing network lifecycle.

The lifecycle and associated purposes are described by a data structure denoted here as the 'self-growing descriptor' [6]. It is utilized as a descriptive data structure associated with a dedicated entity (node or network, e.g. as an electronic datasheet, or as a capability set) or can be communicated in part or in complete between entities involved, requesting certain behaviour (e.g. as a configuration parameter set) by an entity. When used in communication the self-growing descriptor may be partially communicated:

- It may be merged with existing information by the receiving entity optimizing, for example the communication overhead by avoiding repeated transmission of the same contents, chaining subsequent communication messages each carrying distinct parts of the descriptor;

- It may augment or replace existing information at the receiving entity and may update existing descriptors, for example;

Additionally, descriptors may be modified by certain commands (including via protocol messages or by manual interaction), referencing uniquely identifiable elements of the descriptor such as parameters or rules and removing, disabling or enabling these elements. This capability also characterises a self-growing descriptor as a configuration parameter set. Hence, a self-growing descriptor as well as its key elements must be uniquely identifiable (both in a local and global context if needed) and must allow to be bound with a certain device, device class, network, network class, or application, in general setting the applicable scope of uniqueness of a descriptor. If two or more nodes or networks join in a self-growing collaboration, a newly generated (e.g. merged) und unique self-growing descriptor may replace (i.e. temporarily overlay, since recovering from a purpose change may be required) the descriptors of the joining nodes or networks.

The 'Dictionary' element serves two main purposes. First, it describes the attributes and capacities of the entity (i.e. the node, collection of nodes, or network, or compositional parts e.g. a protocol) in terms of realisable parameters and rules. It also provides a searchable database suitable for comparing node or networks for compatibility prior to joining in a self-growing manner. The Dictionary element is designed to enable decision-making, serving as a part of the ontology of the cognitive engines realising decisions. Thus, merging of dictionaries is a common operation in self-growing. The Dictionary is defining possible purposes and progressions in a parameterized form. 
That is, it specifies configuration parameters, but does not specify the values of these parameters. This is achieved by providing a LifeCycle element.

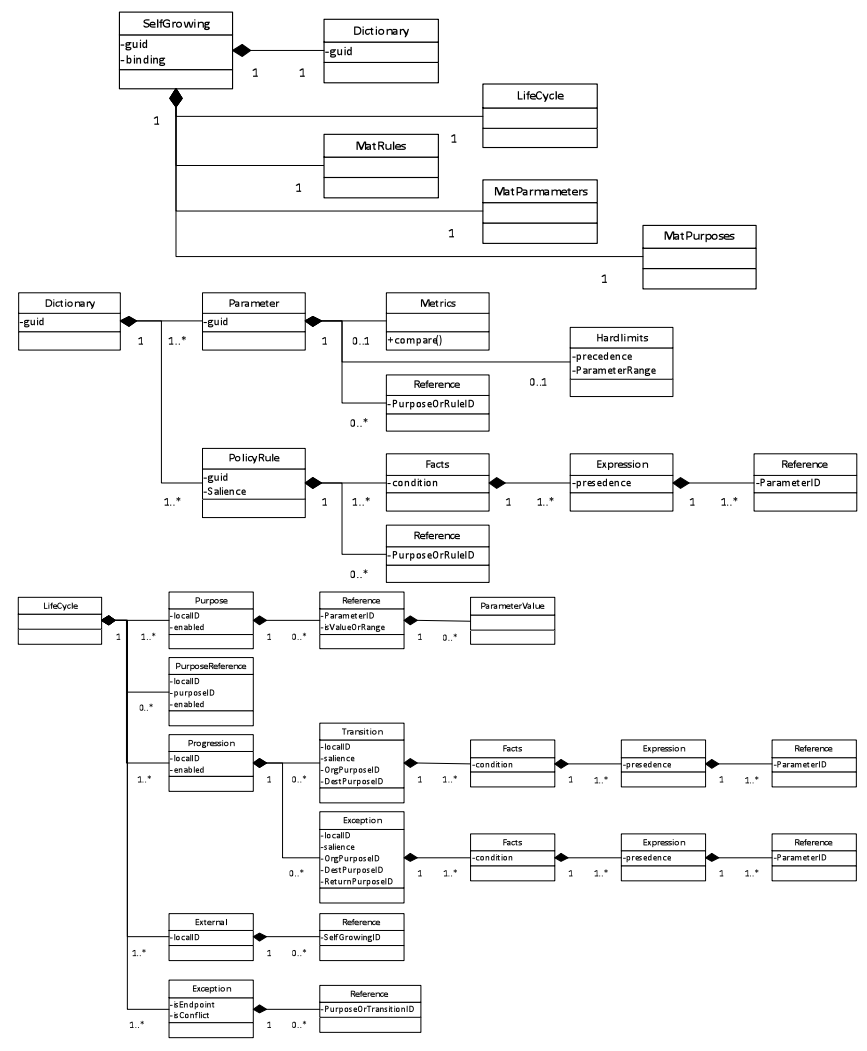

Figure III-2: Self-growing descriptor: top-level data structure.

The 'LifeCycle' element links the Parameters and PolicyRules summarized by the Dictionary element and specifies the self-growing attribute of the associated entity by defining attainable purposes and progressions. In addition, it can reference local rules, parameters or purposes provided by the 'MatRules', 'MatPurposes' and 'MatParameters' elements to include self-learned attributes into a lifecycle, for example. In addition, the LifeCycle element defines Purposes by giving associated Parameters and assigning values to these parameters. The resulting set of parameter values defines the configuration required to realise a target purpose for the given entity. It may also reference learned or dynamically created purposes.

Since not all potential configurations a network can assume during its lifecycle are matching a dedicated purpose, these configuration states herein are denoted as 'Progression Points'. How a self-growing network evolves between progression points shall be defined by suitable rules. Hence, the evolution of a self-growing network through its lifecycle can be described by a non-empty set of rules. Applying a rule may cause a change in attributes or parameters when commuting between progression points. The benefit of applying a certain rule is measurable, given that both the starting point and the endpoint of a transition between progression points are well-defined and are measurable.
Rules might be static (e.g. known a-priori), volatile or dynamic (e.g. computed), or persistent (e.g. self-learned). Figure III-3 provides an example for the top-level rules of the lifecycle example shown in Figure III-1. Note that in an inference engine implementation, firing a rule in a timely manner implicitly requires an external trigger, or programmed request not shown here, except for transitional progression points which are assumed to be self-triggered.

When defining or optimizing a lifecycle, the benefit of a purpose and the cost of a progression can be utilized as a metric. Assuming backward reasoning as a suitable method (without limiting further realisations), we can describe the cost of applying a rule as $\operatorname{cost}(A \rightarrow B)$ to attain a new purpose $B$ inferred from a purpose $A$ under some external triggers, facts and conditions (cf. Figure III-3).

Thus, the additional cost of handling an event by the network is

$$
\begin{array}{r}
\operatorname{cost}(\text { Event } \wedge \text { Recover })=\operatorname{cost}(E \wedge \text { Recover } \rightarrow D)+ \\
\operatorname{cost}(B \wedge \text { Event } \rightarrow E)-\operatorname{cost}(B \wedge \neg \text { Event } \rightarrow D)
\end{array}
$$

While the minimum cost to reach purpose $\mathrm{G}$ is: $\operatorname{cost}(B \wedge \neg$ Event $\rightarrow D)+\operatorname{cost}(D \wedge$ Decision $\rightarrow G)$.

The cost of approaching $G$ and recovering first from the event-driven reconfiguration is $\operatorname{cost}(B \wedge$ Event $\rightarrow E)+\operatorname{cost}(E \wedge$ Recover $\wedge$ Decision $\rightarrow G)$.

Omitting complete recovery and approaching a matched purpose (that probably only recovers partially since omitting $\mathrm{D}$ and applying $\mathrm{C}$ instead, which according to the rules given is the only way to reach $G$ without applying Recover) is $\operatorname{cost}(B \wedge$ Event $\rightarrow E)+\operatorname{cost}(E \wedge \neg$ Recover $\wedge$ Decision $\rightarrow G)$, which might be more beneficial in the long run.

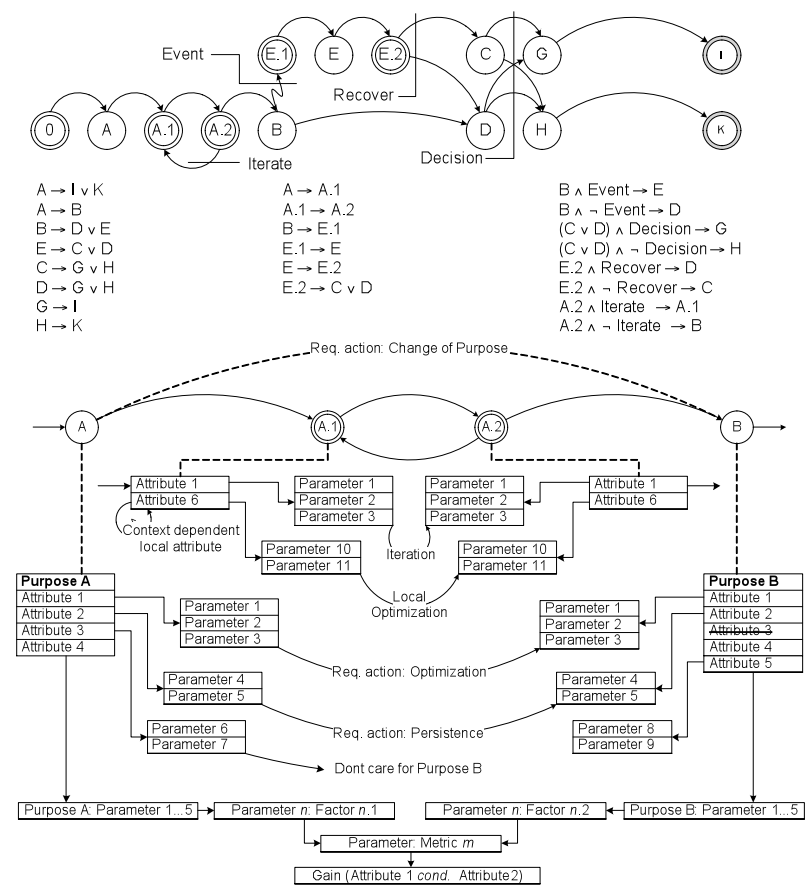

Figure III-3: Sample progression between purposes of a selfgrowing network through transient configurations. 


\section{COGNITIVE ARCHITECTURE}

A full self-growing architecture (cf. Figure IV-1 for the logical architecture) consists of reconfigurable nodes and / or networks in that nodes and / or networks associate with (potentially collocated) cognitive decision-making capacity, which in turn controls collocated or distributed functions (implementing reconfiguration capacity) via the $\mathrm{CCE}-\mathrm{CF}$ interface between a CONSERN cognitive engine (CCE) and a CONSERN enabled function (CF). The CONSERN entity (CE) is realizing a CCE and may enable a collocated legacy function to interface with a CCE.

CONSERN enabled network elements (denoted further as CONSERN entities for simplicity) provide self-organizing and self-growing capabilities as specified further in [6]. The Managed Resource models a physical network node, which may be configurable via legacy interfaces but is not a $\mathrm{CE}$ on its own. The interconnection of a legacy network (facilitating network nodes that are not CEs) with CEs is realized through the CONSERN Configurable Gateway (CCG). The CONSERN Sensor Coordinator (CSC) and Sensor Data Aggregator (which is an external / legacy entity on its own) enable the coordination and control of collocated sensor networks by CEs. Finally, the CONSERN Policy Manager (CPM) is introduced to translate high-level business or regulatory goals injected by the Policy Provider to specific policy rules that will be enforced to the CEs and, potentially, to legacy network elements through a CE.

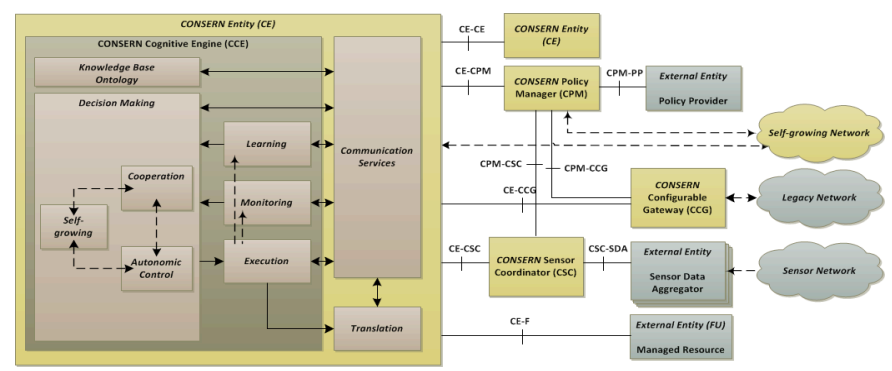

Figure IV-1: Full self-growing logical architecture.

Distributed cognitive engines are communicating and coordinating utilizing the CCE-CCE interface to control the self-growing capacity of the compound system. In addition, a focussed CCE-CCE interface is realized to enable coexistence, coordination and collaboration between selfgrowing systems, potentially exchanging information required to prepare and initiate merging of these selfgrowing systems into a single system.

The cognitive portion of a full self-growing architecture enables planning and decision-making of purposes and life cycles in 'it's own network', can coordinate with neighbouring networks, and can evaluate and judge upon potential benefits of cooperating or joining with neighbouring networks. In addition it may detect potential conflicts in purposes or life cycles prior to initiate collaboration between networks (cf. Figure IV-2).

From the discussion above a stratum model can be developed resembling that given earlier for UMTS [7] (cf. Figure IV-3). The purpose of this model here is to depict the interaction (in terms of information exchange across interfaces) of cognitive engines in the CONSERN logical architecture. For the stratum model it is assumed that cognitive decision-making is incorporated either as a cognitive function with a network node or with a dedicated node hosting a decision engine (e.g. as a dedicated service node). The latter opens up a potential migration path to enhance existing systems by self-growing capacity.

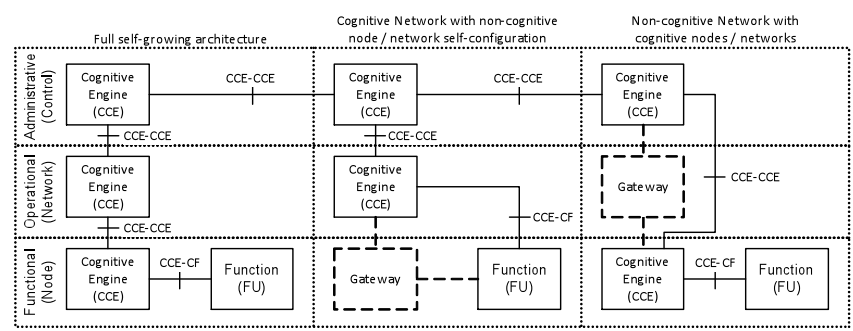

Logical Interface Physical Interface

Figure IV-2: Logical model and basic applications of a selfgrowing architecture to various topologies.

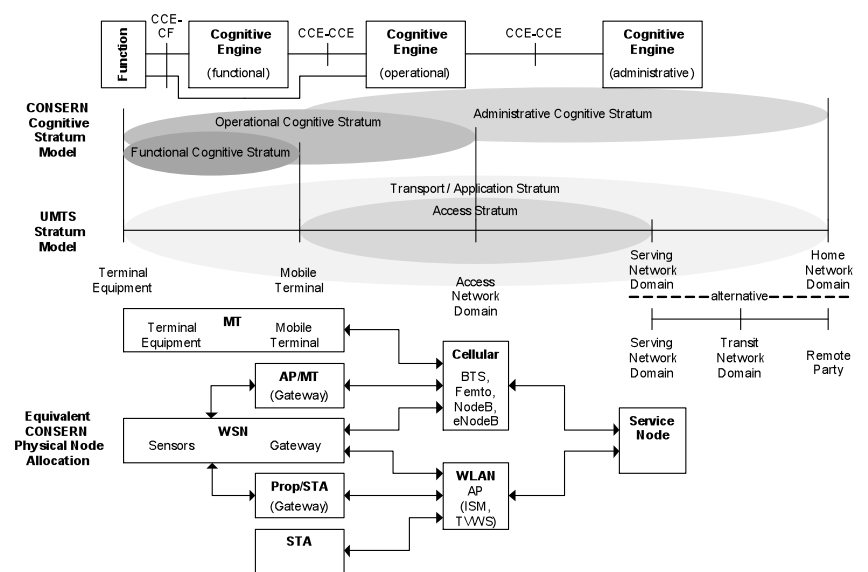

Figure IV-3: Stratum model of the self-growing cognitive architecture

\section{USE CASES AND APPLICATION EXAMPLES}

In the context of the EU FP7 CONSERN project, there have been defined sixteen use cases each of them requires different levels of self-growing capabilities by the system. As already described, a 'full self-growing system' supports self-growing capabilities at the administrative level, at the operational level, and at the functional (node) level.

Based on the description and the analysis of the use cases given in detail in [4], four of them are seen as best suited to highlight the benefit self-growing and to simultaneously demonstrate full self-growing capabilities [6]:

- Energy optimization in an office environment under coverage constraints, emphasizing on scalability and coexistence issues in a dense environment;

- Energy-aware end-to-end delay optimization, emphasizing on purpose changes due to dynamic collaboration goals;

- $\quad$ Purpose-driven network reconfiguration during an emergency situation, emphasizing on event-driven 
reconfigurations across layers, involving nodes and heterogeneous network collaboration;

- Dynamic Meeting Setup in Flexible Office/Building Environments, emphasizing on coexistence and lifecycle management in the presence of multiple simultaneous purpose changes over time.

In the following sections, examples of the application of the proposed cognitive architecture are presented for two of the above use cases.

\section{A. Applying the self-growing architecture to 'Energy optimization in an office environment under coverage constraints '}

A dense environment is considered in which several WiFi APs and UMTS femtocells are collocated and the use of sensors enables controlling the network elements' operation in order to achieve energy efficiency under coverage constraints. This can be based for example on keeping specific network elements operational only when needed. This enables the network operator to identify which is the optimum network configuration regarding the users' needs in the corresponding area.

The self-growing paradigm is triggered, when the presence of a person(s) is sensed by the WSN in a specific room of the building; at the same time, user presence is also identified through a network event (e.g. the terminal, or UE, is associated with a WiFi AP).

The following steps are executed by the different entities of the proposed CONSERN architecture:

1. The CONSERN Entity $(\mathrm{CE})$ that is integrated in the network element (which is controlling the macrocell), collects and combines information from the WSN (e.g. motion detected in an area) by invoking the Sensor Data Aggregator and the CONSERN Sensor Coordinator, and the network element (e.g., WLAN association),

2. The Decision Making function of the CE decides on whether it is preferable (for example with regards to energy constraints) to switch-on another AP or a femtocell (i.e. a Network Element - NE) that is closer to the UE rather than keep serving it from the current NE,

3. According to the decision, the CE notifies through the Communication Services function the NE which is closer to the UE about the decision made on the preferable change on the operation status,

4. The NE that is closer to the UE evaluates the notification under its own context and the network operator's policies, and, in case, changes its operational status,

5. The NE that is currently serving the UE may reduce its transmission power upon the activation of the $\mathrm{NE}$ that is closer to the UE,

6. If the UE is a CONSERN-enabled device, it is notified to handoff to the new NE (AP or femtocell). If it is not a CONSERN-enabled device, the presence of the new NE combined with the lower SNR by the serving NE will drive the UE to associate to the new $\mathrm{NE}$ that is located near the UE,
7. The CE communicates (Communication Services function) and collects information from neighbouring network elements (Monitoring function) to optimise the operation of the APs and the femtocells (e.g. collecting information required for the proper channel allocation) in order to maximize the average time before communication interruption.

\section{B. Applying self-growing architecture to 'Purpose-driven network reconfiguration during an emergency situation '}

Sensor nodes forming an ad-hoc network are deployed in a given environment partially covered by a second type of network providing centralized, single-hop backbone access, e.g. IEEE 802.11 WLAN. Both networks had gone through the self-growing phase having resulted in an integrated, symbiotic network under the control of cognitive decision entities. Under normal operation, the sensor network provides sensing information (e.g. temperature in various locations of a building) at low duty cycles; the network is optimized for long network lifetime accepting higher delays in the acquisition of sensing information. An incident situation occurs (e.g. a fire in parts of a building). As a result, parts of the existing sensor node infrastructure are destroyed. Also, as a result of the incident situation, the metric driving the network configuration changes and the network has to fulfil a new purpose: an extremely long lifetime of the network is less important but sensing information has to be delivered in a timely manner. Besides, the sensor nodes have to additionally detect persons in the building or even track the movement of emergency responders. For such, coverage in specific areas has to be ensured. Cognitive decision engines detect this situation and decide on a network reconfiguration, possibly including the symbiotic effects of the integrated WLAN network to cope with the incident situation and its associated change in purpose.

Indication of incident situation resulting in a change of the target purpose is the event that will trigger the realization of the self-growing paradigm.

The following steps are executed by the different entities of the proposed CONSERN architecture:

1. Contextual information delivered either by an existing WSN (through Sensor Data Aggregator(s) and CONSERN Sensor Coordinators) or CONSERN-enabled NEs (through the Monitoring and Communication Services functions) trigger the change of purpose of the existing CEs,

2. Information exchange takes place among the CEs to identify the new purpose and update their Knowledge Base Ontologies with the necessary information,

3. The Decision Making function identifies available nodes as well as potentially available links between nodes (of collocated networks),

4. The Execution function through the Translation function enforces the reconfiguration of nodes enabling the new purpose, 
5. Information exchange is performed through the Communication Services function and/or the CONSERN Configurable Gateway to achieve crossnetwork optimization of active communication paths and node usage under coverage, energy and network lifetime constraints.

This process is ended when one of the following conditions is reached:

- The target purpose is achieved for the first time (i.e. the network offers the required functionality),

- The CONSERN Cognitive Engine decides that it is impossible to achieve the target purpose,

- The target purpose changes.

Conclusions from the application of the self-growing architecture on the use cases are presented and discussed in more detail in [4][6].

\section{EXPECTED BENEFITS AND OUTLOOK}

An detailed evaluation of the self-growing paradigm from a technological and business perspective is under way in the course of the EU FP7 CONSERN project. This section thus capitalises on preliminary findings regarding the utilisation of the self-growing paradigm as a new emerging technology for the future flexible and trustworthy network architecture. The discussion covers three levels of results, namely, (i) results on general architectural requirements attributed to the design principles of the self-growing paradigm; (ii) results on the applicability of the proposed architecture on specific use cases; and (iii) outlook for next steps in the development of the architecture.

One of the key design requirements for Future Internet is trustworthiness which can be broadly defined as a requirement encompassing dependability and security. Other design requirements such as scalability, openness, evolvability and economic viability, are considered as being fundamental. The self-growing paradigm addresses all the above requirements by utilising a purpose-driven cognitive process performed cooperatively between cognitive engines representing network entities. The cognitive entities can communicate their attributes and capacities to each other and participate in distributed decision-making process where each cognitive entity may uniquely contribute to achieve a given purpose. Dependability is inherent to the self-growing paradigm as it facilitates reconfigurability of the network in a controlled manner by means of rules as provided by the operator. This is significantly different from previous paradigms as it allows the network to optimise its operations while it facilitates the control and the orchestration of its operation.

As illustrated by the use cases the self-growing paradigm extends the self-organising paradigm in two ways. First, it provides the primitives required for the optimisation of multiple purposes in parallel or in sequence as regulated by the lifecycle, the operator's priorities and/or other (external or environmental) circumstances and events. Second, it allows the planning of the utilisation of network entities for the achievement of a purpose for which they have not been primarily designed. Diverse types of networks may be utilised for many purposes that otherwise would require a dedicated network solution. This turns the self-growing paradigm into an economic viable one because the same networks nodes and entities can be reused in various ways and for various purposes.

Implementing the above architecture is associated with communication related overhead costs pertinent to information exchange and control synchronisation. Also allowing cooperation between different administrative authorities could be one of the challenges to be addressed as it requires an extension of the dependability to include incentives for the fulfilment of commitments towards a purpose.

\section{CONCLUSION}

This paper introduced a novel cognitive architecture based on the self-growing paradigm as developed within the EU FP7 CONSERN project. To this end, the fundamental architectural principles and the requirements to be satisfied by the proposed comprehensive architecture have been discussed and its application in two use cases has been demonstrated. The self-growing architecture proposed in this paper and the underlying design principles and architectural components can provide a foundation for flexible, open and trustworthy networks; aspects such as trustworthiness are subject for further evaluation of the concept.

\section{ACKNOWLEDGMENT}

The research leading to these results has received funding from the European Community's Seventh Framework Programme (FP7/2007-2013) under grant agreement CONSERN n ${ }^{\circ} 257542$.

\section{REFERENCES}

[1] INFSO-ICT-257542 CONSERN Project, http://www.ict-consern.eu

[2] N. Alonistioti, A. Merentitis, M. Stamatelatos, E. Schulz, C. Zhou, G. Koudouridis, B. Bochow, M. Schuster, P. Demeester, P. Ballon, S. Delaere, M. Mueck, C. Drewes, L. V. der Perre, J. Declerck, T. Lewis, and I. Chochliouros, 'Towards self-adaptable, scalable, dependable and energy efficient networks: The self-growing concept', in UBICOMM 2010, 2010.

[3] B. Bochow, M. Schuster, L. Thiem, and J. Tiemann, "A novel system paradigm for self growing wireless networks," 2010. [Online]. Available: http://publica.fraunhofer.de/documents/N-132320.html

[4] CONSERN Deliverable D1.1, 'Scenarios, Use Cases, and System Requirements'

[5] CONSERN Deliverable D4.1, 'Initial Description of Self-Growing Scenarios, Properties, Requirements and Envisaged Framework'.

[6] CONSERN Deliverable D4.2, 'Distributed Self-Growing Architecture and Interface Description'.

[7] 3GPP TS 23.101 V9.0.0 (2009-12), "3rd Generation Partnership Project; Technical Specification Group Services and System Aspects, General Universal Mobile Telecommunications System (UMTS) architecture (Release 9)". 